\title{
Sewage sludge gasification in a fluidized bed: experimental
} investigation and modeling

4 Renata Migliaccio ${ }^{\mathrm{a}}$, Paola Brachi* ${ }^{*}$, Fabio Montagnaro $^{\mathrm{b}}$, Salvatore Papa ${ }^{\mathrm{c}}$, Alberto Tavano ${ }^{\mathrm{c}}$,

6

7

${ }^{a}$ Istituto di Scienze e Tecnologie per l'Energia e la Mobilità Sostenibili, Consiglio Nazionale delle Ricerche (STEMS-CNR) Piazzale V. Tecchio 80, 80125 Napoli, Italy Monte Sant'Angelo, 80126 Napoli, Italy 

analysis $(\mathrm{dsb}=\mathrm{dry}$ sludge basis; $\mathrm{dab}=\mathrm{dry}$ and ash free basis).

\begin{tabular}{|c|c|c|c|c|}
\hline \multirow[b]{2}{*}{ Element } & \multicolumn{2}{|c|}{ SS-A } & \multicolumn{2}{|c|}{ SS-B } \\
\hline & $\mathrm{mg} / \mathrm{kg}$ dsb & mg/kg dab & $\mathrm{mg} / \mathrm{kg} \mathrm{dsb}$ & $\mathrm{mg} / \mathrm{kg}$ dab \\
\hline $\mathrm{Ca}$ & 38111 & 112091 & 26133 & 94456 \\
\hline $\mathrm{Al}$ & 11122 & 32711 & 8049 & 29093 \\
\hline $\mathrm{P}$ & 10828 & 31847 & 9942 & 35935 \\
\hline $\mathrm{Fe}$ & 9071 & 26679 & 6008 & 21716 \\
\hline $\mathrm{Si}$ & 6918 & 20347 & 3476 & 12564 \\
\hline $\mathrm{K}$ & 5257 & 15461 & 2922 & 10561 \\
\hline $\mathrm{Mg}$ & 4023 & 11832 & 2588 & 9354 \\
\hline $\mathrm{Na}$ & n.d. & n.d. & 980.1 & 3542 \\
\hline $\mathrm{Zn}$ & 560.1 & 1647 & 731.2 & 2643 \\
\hline $\mathrm{Ti}$ & 434.7 & 1278 & 263.6 & 952.8 \\
\hline $\mathrm{Ba}$ & 194.3 & 571.5 & n.d. & n.d. \\
\hline $\mathrm{Cu}$ & 190.8 & 561.2 & 222.4 & 803.9 \\
\hline Mn & 118.4 & 348.2 & 75.90 & 274.3 \\
\hline $\mathrm{Sr}$ & 76.93 & 226.3 & 195.1 & 705.2 \\
\hline $\mathrm{Pb}$ & 63.88 & 187.9 & n.d. & n.d. \\
\hline $\mathrm{Cr}$ & 28.57 & 84.03 & 28.40 & 102.6 \\
\hline $\mathrm{V}$ & 26.10 & 76.76 & 21.13 & 76.37 \\
\hline $\mathrm{Zr}$ & 12.12 & 35.65 & 53.00 & 191.6 \\
\hline $\mathrm{Ni}$ & 11.97 & 35.20 & 13.16 & 47.57 \\
\hline $\mathrm{Ga}$ & n.d. & n.d. & 26.48 & 95.71 \\
\hline $\mathrm{Li}$ & 8.92 & 26.24 & 2.77 & 10.01 \\
\hline As & n.d. & n.d. & 22.93 & 82.88 \\
\hline Mo & 6.80 & 20.00 & 7.20 & 26.02 \\
\hline $\mathrm{Pd}$ & n.d. & n.d. & 1.75 & 6.32 \\
\hline $\mathrm{Sb}$ & 2.88 & 8.47 & 2.46 & 8.89 \\
\hline Co & 2.28 & 6.71 & 1.78 & 6.43 \\
\hline Sn & 0.16 & 0.47 & n.d. & n.d. \\
\hline $\mathrm{Ag}$ & n.d. & n.d. & 6.23 & 22.52 \\
\hline $\mathrm{Se}$ & n.d. & n.d. & 2.91 & 10.52 \\
\hline $\mathrm{Cd}$ & n.d. & n.d. & 0.66 & 2.39 \\
\hline $\mathrm{Be}$ & n.d. & n.d. & 0.37 & 1.34 \\
\hline
\end{tabular}

Table S1. Elemental composition for parent SS-A and SS-B samples as detected by ICP-MS 
19 Table S2. Trace elements detected in SS-A by EDX analysis (companion of Figure 2 in the 20 Manuscript). Data in mass percentage.

\begin{tabular}{lc}
\hline \multicolumn{1}{r}{ Element } & \% \\
\hline $\mathrm{O}$ & 83.01 \\
$\mathrm{Ca}$ & 5.70 \\
$\mathrm{Si}$ & 4.65 \\
$\mathrm{Fe}$ & 1.61 \\
$\mathrm{Al}$ & 1.61 \\
$\mathrm{~K}$ & 1.30 \\
$\mathrm{~S}$ & 1.22 \\
$\mathrm{P}$ & 0.91 \\
\hline
\end{tabular}

21 
Table S3. Semi-quantitative composition of tar (GC-MS analysis) collected in the two condensation stages during FB gasification of SS-A.

\begin{tabular}{|c|c|c|c|c|}
\hline \multirow[b]{2}{*}{ Component } & \multicolumn{2}{|c|}{$\begin{array}{c}\text { Non-refrigerated } \\
\text { condensation stage } \\
\text { (room temperature) }\end{array}$} & \multicolumn{2}{|c|}{$\begin{array}{c}\text { Refrigerated condensation } \\
\text { stage } \\
\left(-12^{\circ} \mathrm{C}\right)\end{array}$} \\
\hline & $\begin{array}{c}\text { Normalized } \\
\text { peak area, } \\
\%\end{array}$ & Retention time & $\begin{array}{c}\text { Normalized } \\
\text { peak area, } \\
\%\end{array}$ & Retention time \\
\hline Nitrogen compounds & & & & \\
\hline Pyridine $\left(\mathrm{C}_{5} \mathrm{H}_{5} \mathrm{~N}\right)$ & & & 2.57 & 14.10 \\
\hline Aniline $\left(\mathrm{C}_{6} \mathrm{H}_{7} \mathrm{~N}\right)$ & & & 1.64 & 29.71 \\
\hline 3-Pyridinecarbonitrile $\left(\mathrm{C}_{6} \mathrm{H}_{4} \mathrm{~N}_{2}\right)$ & & & 1.64 & 31.59 \\
\hline $\begin{array}{l}\text { 2,2,6,6-Tetramethyl-4-piperidone } \\
\left(\mathrm{C}_{9} \mathrm{H}_{17} \mathrm{NO}\right)\end{array}$ & 16.99 & 37.05 & 28.97 & 37.04 \\
\hline Isoquinoline $\left(\mathrm{C}_{9} \mathrm{H}_{7} \mathrm{~N}\right)$ & 4.74 & 42.84 & & \\
\hline Quinoline $\left(\mathrm{C}_{9} \mathrm{H}_{7} \mathrm{~N}\right)$ & 1.95 & 44.11 & 3.74 & 42.84 \\
\hline Pyrimidine $\left(\mathrm{C}_{4} \mathrm{H}_{4} \mathrm{~N}_{2}\right)$ & & & 1.17 & 14.77 \\
\hline Indole $\left(\mathrm{C}_{8} \mathrm{H}_{7} \mathrm{~N}\right)$ & 7.24 & 48.07 & 3.74 & 48.07 \\
\hline 2-Cyanonaphthalene $\left(\mathrm{C}_{11} \mathrm{H}_{7} \mathrm{~N}\right)$ & 4.18 & 55.65 & & \\
\hline 1-Cyanonaphthalene $\left(\mathrm{C}_{11} \mathrm{H}_{7} \mathrm{~N}\right)$ & 3.62 & 57.02 & 1.17 & 55.65 \\
\hline Ketones & & & & \\
\hline 4-Methyl-3-penten-2-one $\left(\mathrm{C}_{6} \mathrm{H}_{10} \mathrm{O}\right)$ & & & 2.34 & 17.34 \\
\hline $\begin{array}{l}\text { 4-Hydroxy-4-methyl-2-pentanone } \\
\left(\mathrm{C}_{6} \mathrm{H}_{12} \mathrm{O}_{2}\right)\end{array}$ & & & 33.88 & 21.67 \\
\hline Phenolic compounds & & & & \\
\hline Phenol $\left(\mathrm{C}_{6} \mathrm{H}_{6} \mathrm{O}\right)$ & & & 1.17 & 32.61 \\
\hline Aromatic compounds & & & & \\
\hline Biphenyl $\left(\mathrm{C}_{12} \mathrm{H}_{10}\right)$ & 3.06 & 48.61 & 1.40 & 48.61 \\
\hline PAH & & & & \\
\hline Benzocycloheptatriene $\left(\mathrm{C}_{11} \mathrm{H}_{10}\right)$ & & & 1.40 & 45.78 \\
\hline Naphthalene $\left(\mathrm{C}_{10} \mathrm{H}_{8}\right)$ & 7.24 & 39.54 & 4.21 & 39.53 \\
\hline 1-Methylnaphthalene $\left(\mathrm{C}_{11} \mathrm{H}_{10}\right)$ & 1.95 & 44.96 & & \\
\hline 2-Methylnaphthalene $\left(\mathrm{C}_{11} \mathrm{H}_{10}\right)$ & 2.23 & 45.79 & 1.17 & 44.96 \\
\hline 2-Vinylnaphthalene $\left(\mathrm{C}_{12} \mathrm{H}_{10}\right)$ & 3.34 & 51.27 & & \\
\hline Acenaphthene $\left(\mathrm{C}_{12} \mathrm{H}_{10}\right)$ & & & 1.17 & 51.26 \\
\hline Acenaphthylene $\left(\mathrm{C}_{12} \mathrm{H}_{8}\right)$ & 11.42 & 52.61 & 4.91 & 52.61 \\
\hline Fluorene $\left(\mathrm{C}_{13} \mathrm{H}_{10}\right)$ & 7.81 & 57.97 & & \\
\hline Anthracene $\left(\mathrm{C}_{14} \mathrm{H}_{10}\right)$ & 15.88 & 66.00 & & \\
\hline Phenanthrene $\left(\mathrm{C}_{14} \mathrm{H}_{10}\right)$ & 4.18 & 66.36 & 3.74 & 65.99 \\
\hline Pyrene $\left(\mathrm{C}_{16} \mathrm{H}_{10}\right)$ & 4.18 & 82.64 & & \\
\hline
\end{tabular}


Table S4. Semi-quantitative composition of tar (GC-MS analysis) collected in the two condensation stages during FB gasification of SS-B.

\begin{tabular}{|c|c|c|c|c|}
\hline \multirow[b]{2}{*}{ Component } & \multicolumn{2}{|c|}{$\begin{array}{c}\text { Non-refrigerated } \\
\text { condensation stage } \\
\text { (room temperature) }\end{array}$} & \multicolumn{2}{|c|}{$\begin{array}{c}\text { Refrigerated condensation stage } \\
\left(-12{ }^{\circ} \mathrm{C}\right)\end{array}$} \\
\hline & $\begin{array}{c}\text { Normalized } \\
\text { peak area, } \%\end{array}$ & $\begin{array}{c}\text { Retention } \\
\text { time }\end{array}$ & $\begin{array}{c}\text { Normalized } \\
\text { peak area, } \%\end{array}$ & $\begin{array}{c}\text { Retention } \\
\text { time }\end{array}$ \\
\hline Nitrogen compounds & & & & \\
\hline Pyridine $\left(\mathrm{C}_{5} \mathrm{H}_{5} \mathrm{~N}\right)$ & & & 1.05 & 14.03 \\
\hline Benzonitrile $\left(\mathrm{C}_{7} \mathrm{H}_{5} \mathrm{~N}\right)$ & & & 2.33 & 29.89 \\
\hline $\begin{array}{l}\text { 2,2,6,6-Tetramethyl-4-piperidone } \\
\left(\mathrm{C}_{9} \mathrm{H}_{17} \mathrm{NO}\right)\end{array}$ & 3.40 & 37.00 & & \\
\hline Isoquinoline $\left(\mathrm{C}_{9} \mathrm{H}_{7} \mathrm{~N}\right)$ & 3.23 & 42.79 & 1.20 & 44.04 \\
\hline Quinoline $\left(\mathrm{C}_{9} \mathrm{H}_{7} \mathrm{~N}\right)$ & 1.49 & 44.04 & 42.79 & 42.79 \\
\hline Indole $\left(\mathrm{C}_{8} \mathrm{H}_{7} \mathrm{~N}\right)$ & 5.76 & 48.02 & 3.18 & 48.01 \\
\hline Pyrrole-3-carbonitrile $\left(\mathrm{C}_{5} \mathrm{H}_{4} \mathrm{~N}_{2}\right)$ & 1.79 & 52.14 & & \\
\hline Carbazole $\left(\mathrm{C}_{12} \mathrm{H}_{9} \mathrm{~N}\right)$ & 3.35 & 70.44 & & \\
\hline 1-Cyanonaphthalene $\left(\mathrm{C}_{11} \mathrm{H}_{7} \mathrm{~N}\right)$ & 3.41 & 55.60 & 1.82 & 55.60 \\
\hline 2-Cyanonaphthalene $\left(\mathrm{C}_{11} \mathrm{H}_{7} \mathrm{~N}\right)$ & 3.07 & 56.97 & & \\
\hline Ketones & & & & \\
\hline $\begin{array}{l}\text { 4-Hydroxy-4-methyl-2-pentanone } \\
\left(\mathrm{C}_{6} \mathrm{H}_{12} \mathrm{O}_{2}\right)\end{array}$ & & & 1.51 & 21.62 \\
\hline Aromatic compounds & & & & \\
\hline Biphenyl (C12H10) & 1.39 & 48.58 & 1.54 & 48.57 \\
\hline PAH & & & & \\
\hline Indene $\left(\mathrm{C}_{9} \mathrm{H}_{8}\right)$ & & & 2.74 & 31.49 \\
\hline Naphthalene $\left(\mathrm{C}_{10} \mathrm{H}_{8}\right)$ & 3.03 & 39.50 & 15.07 & 39.50 \\
\hline 2-Methylnaphthalene $\left(\mathrm{C}_{11} \mathrm{H}_{10}\right)$ & 1.43 & 45.75 & 1.92 & 44.92 \\
\hline 1-Methylnaphthalene $\left(\mathrm{C}_{11} \mathrm{H}_{10}\right)$ & & & 1.70 & 45.74 \\
\hline 2-Vinylnaphthalene $\left(\mathrm{C}_{12} \mathrm{H}_{10}\right)$ & 2.17 & 51.24 & 1.39 & 51.23 \\
\hline Acenaphthylene $\left(\mathrm{C}_{12} \mathrm{H}_{8}\right)$ & 6.88 & 52.57 & 4.91 & 52.56 \\
\hline Fluorene $\left(\mathrm{C}_{13} \mathrm{H}_{10}\right)$ & 6.79 & 59.80 & 2.19 & 57.92 \\
\hline Phenanthrene $\left(\mathrm{C}_{14} \mathrm{H}_{10}\right)$ & 12.89 & 65.95 & 2.60 & 65.95 \\
\hline Anthracene $\left(\mathrm{C}_{14} \mathrm{H}_{10}\right)$ & 4.93 & 66.32 & & \\
\hline 4,5-Methylenephenanthrene $\left(\mathrm{C}_{15} \mathrm{H}_{10}\right)$ & 2.66 & 71.16 & & \\
\hline 2-Phenylnaphthalene $\left(\mathrm{C}_{16} \mathrm{H}_{12}\right)$ & 1.67 & 73.06 & & \\
\hline Fluoranthene $\left(\mathrm{C}_{16} \mathrm{H}_{10}\right)$ & 3.78 & 79.13 & & \\
\hline 1,2-Benzanthracene $\left(\mathrm{C}_{18} \mathrm{H}_{12}\right)$ & & & 1.05 & 79.69 \\
\hline Fluoranthene $\left(\mathrm{C}_{16} \mathrm{H}_{10}\right)$ & & & 1.60 & 80.69 \\
\hline Pyrene $\left(\mathrm{C}_{16} \mathrm{H}_{10}\right)$ & 6.78 & 82.55 & 1.74 & 82.54 \\
\hline 2-Methylfluoranthene $\left(\mathrm{C}_{17} \mathrm{H}_{12}\right)$ & 1.27 & 91.20 & & \\
\hline Linear hydrocarbons & & & & \\
\hline Eicosane $\left(\mathrm{C}_{20} \mathrm{H}_{42}\right)$ & 2.19 & 88.44 & & \\
\hline
\end{tabular}


28 Table S5. Elemental composition for bottom ash from fluidized bed gasification of SS-A and 29 SS-B as detected by ICP-MS analysis.

\begin{tabular}{|c|c|c|}
\hline Element & $\begin{array}{l}\text { SS-A ash, } \\
\text { mg/kg dab }\end{array}$ & $\begin{array}{l}\text { SS-B ash, } \\
\text { mg/kg dab }\end{array}$ \\
\hline $\mathrm{Ca}$ & 56649 & 68565 \\
\hline $\mathrm{Al}$ & 17968 & 17492 \\
\hline $\mathrm{Fe}$ & 17424 & 29341 \\
\hline $\mathrm{P}$ & 13623 & 26286 \\
\hline $\mathrm{Mg}$ & 6600 & 6763 \\
\hline $\mathrm{Cu}$ & 5650 & 600 \\
\hline $\mathrm{K}$ & 5440 & 6134 \\
\hline $\mathrm{Si}$ & 3727 & 1890 \\
\hline $\mathrm{Na}$ & 3494 & 3372 \\
\hline $\mathrm{Ti}$ & 990 & 984 \\
\hline $\mathrm{Zn}$ & 394 & 414 \\
\hline $\mathrm{Ba}$ & 259 & n.d. \\
\hline $\mathrm{Mn}$ & 250 & 326 \\
\hline $\mathrm{Sr}$ & 145 & 462 \\
\hline $\mathrm{Cr}$ & 132 & 548 \\
\hline $\mathrm{Ni}$ & 60 & n.d. \\
\hline $\mathrm{V}$ & 52 & 67 \\
\hline $\mathrm{Ga}$ & n.d. & 69 \\
\hline $\mathrm{Pb}$ & 50 & n.d. \\
\hline $\mathrm{Zr}$ & 40 & 32 \\
\hline Mo & 16 & 35 \\
\hline $\mathrm{Sn}$ & 13 & 48 \\
\hline $\mathrm{Li}$ & 8 & 6 \\
\hline $\mathrm{Co}$ & 5 & 6 \\
\hline $\mathrm{Sb}$ & 2 & n.d. \\
\hline $\mathrm{Ag}$ & n.d. & 12 \\
\hline $\mathrm{Se}$ & n.d. & 3 \\
\hline
\end{tabular}


32 Table S6. Trace elements detected in SS-A bottom ash by EDX analysis (companion of Figure

337 in the Manuscript). Data in mass percentage.

\begin{tabular}{lc}
\hline \multicolumn{1}{r}{ Element } & \% \\
\hline $\mathrm{O}$ & 41.29 \\
$\mathrm{Si}$ & 24.58 \\
$\mathrm{Al}$ & 8.84 \\
$\mathrm{Ca}$ & 8.31 \\
$\mathrm{~K}$ & 6.11 \\
$\mathrm{Fe}$ & 5.22 \\
$\mathrm{P}$ & 2.28 \\
$\mathrm{Na}$ & 1.77 \\
$\mathrm{Mg}$ & 0.68 \\
$\mathrm{Ti}$ & 0.58 \\
$\mathrm{~S}$ & 0.33 \\
\hline
\end{tabular}

34 


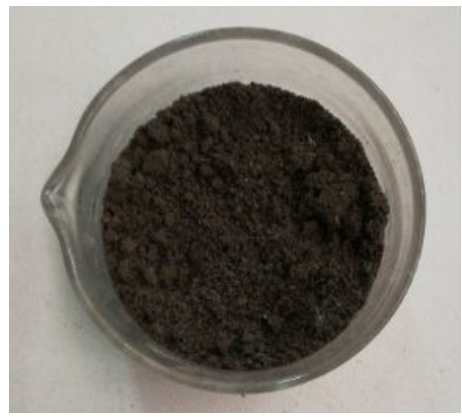

36 Figure S7. Picture of air-dried sewage sludge A (SS-A) sample.

37 

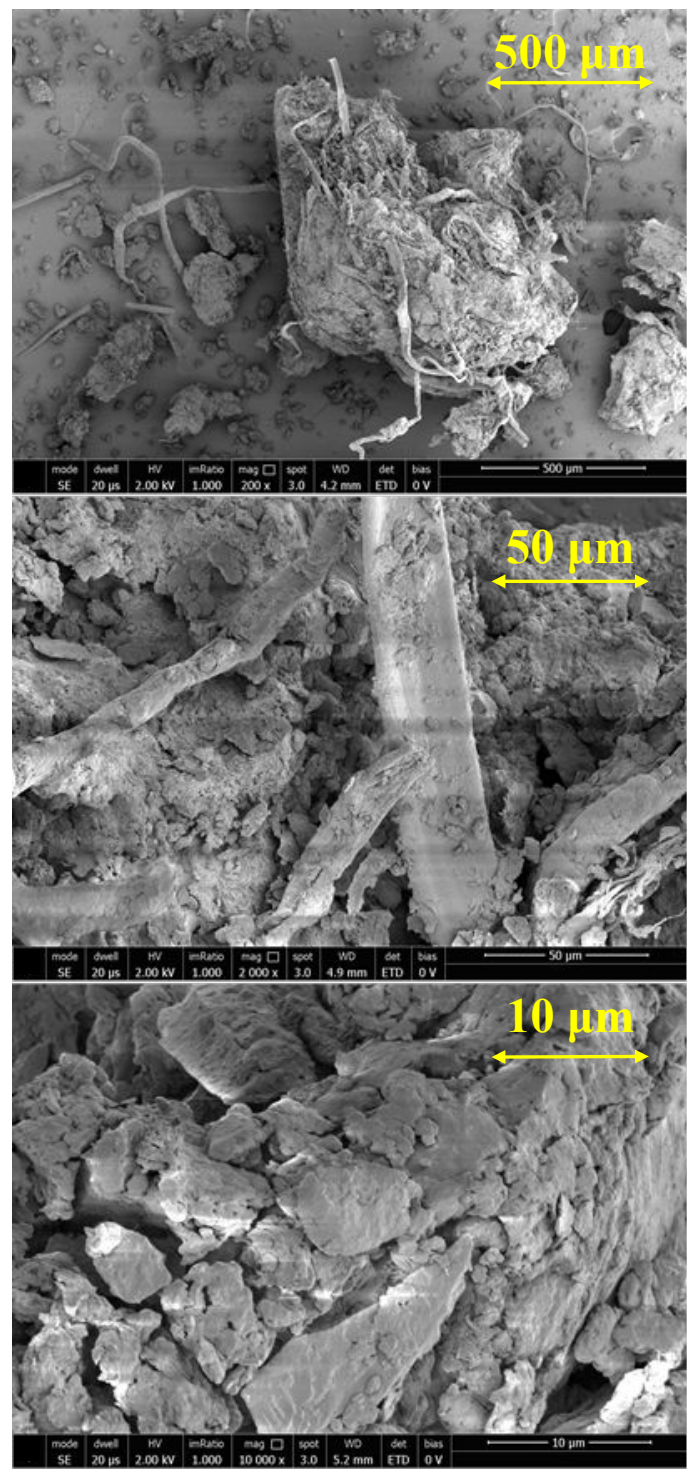

39 Figure S8. SEM images of SS-A (at increasing magnification, from 200 up to $10000 \times$ ). 


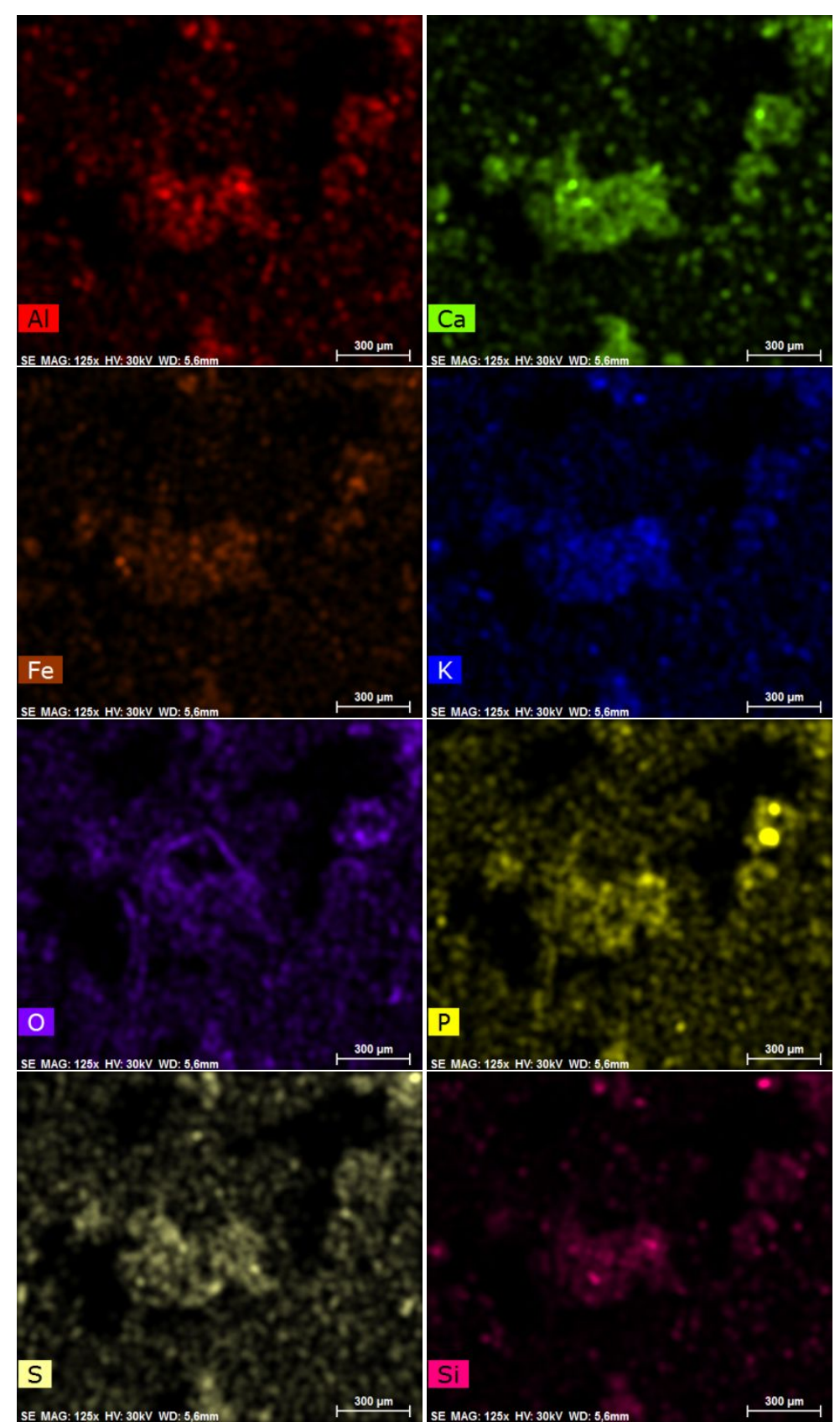

42 Figure S9. Distribution of single elements detected in SS-A (sample of Figure 2 in the 43 Manuscript) by EDX analysis. Position and concentration of each element are identified by 44 false colors and their intensity. 


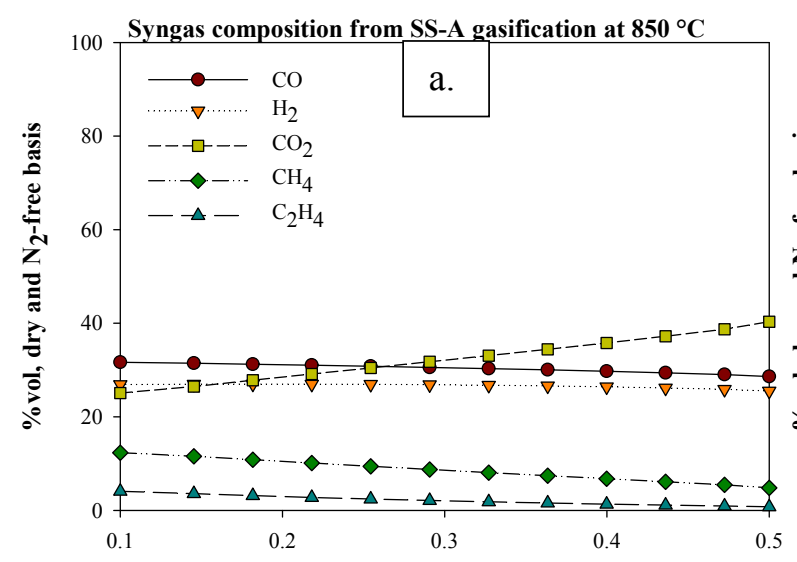

ER, -

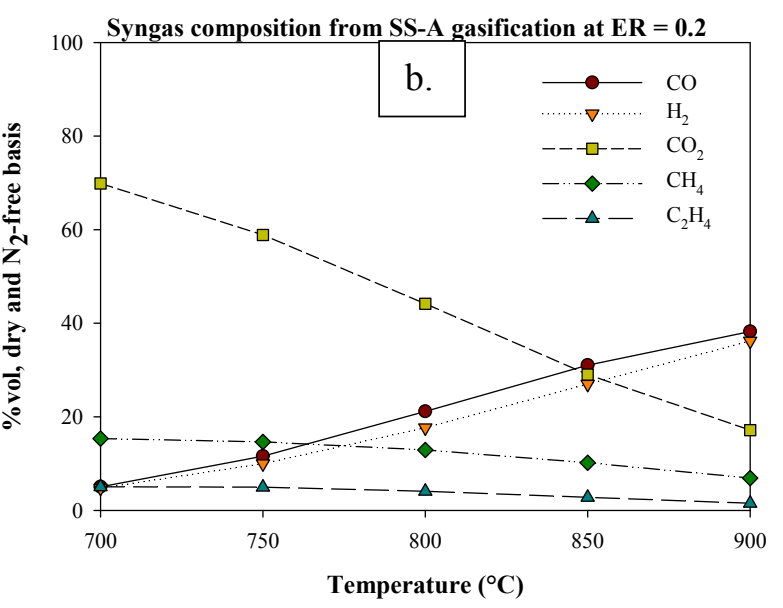

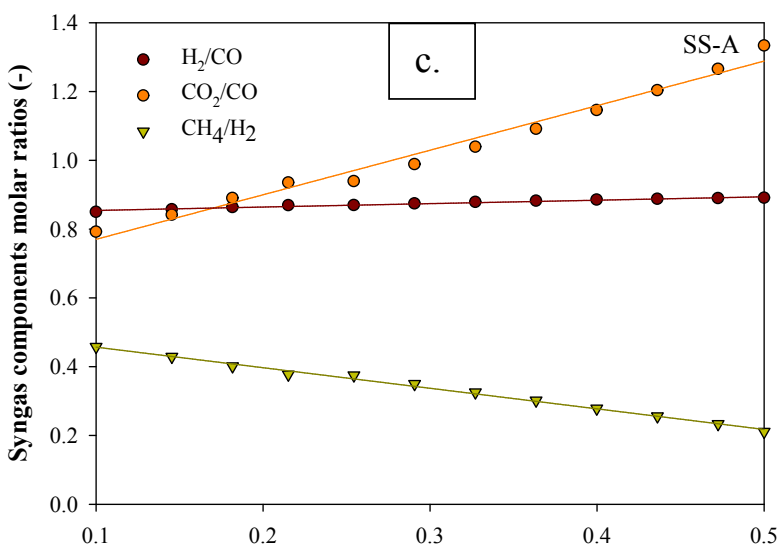

ER, -

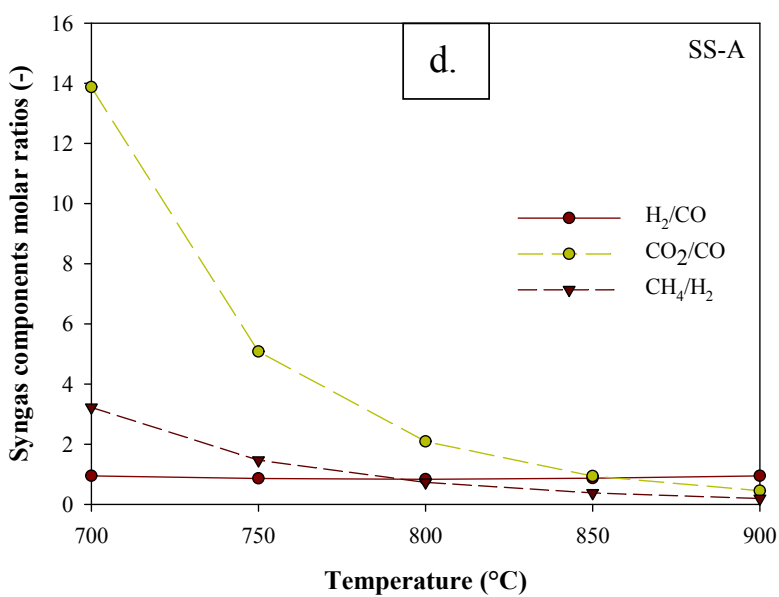

49

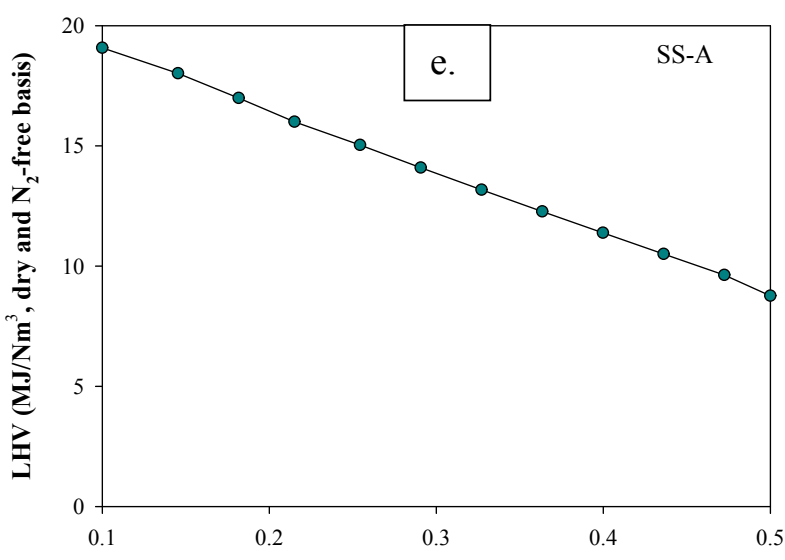

ER, -

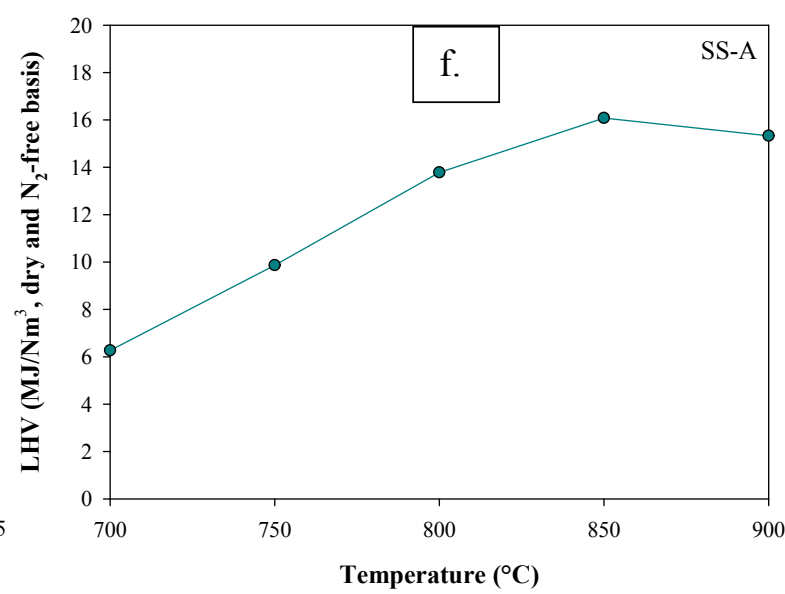

50

Figure S10. Simulated composition, molar ratios and LHV of syngas from gasification of SS-

A at different values of equivalence ratio (a, c and e, respectively, at $850{ }^{\circ} \mathrm{C}$ ) and reactor temperature ( $b, d$ and $f$, respectively, at $E R=0.2$ ). 


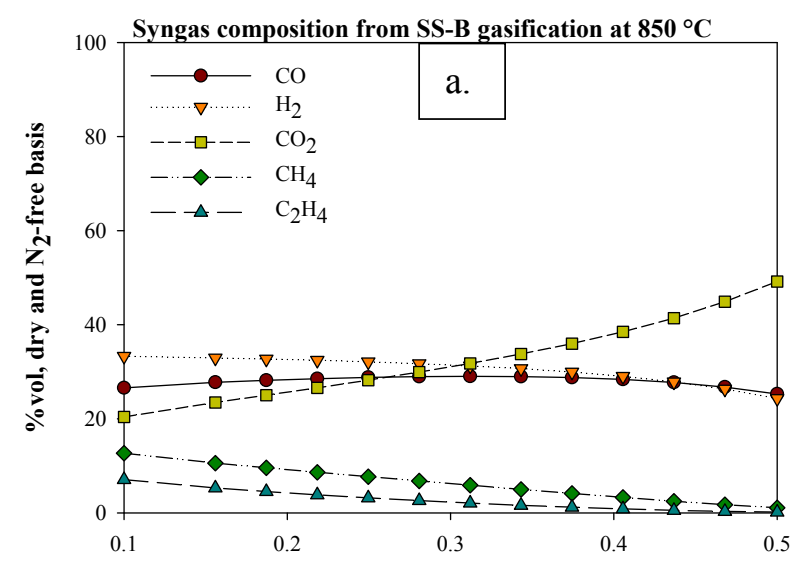

ER, -
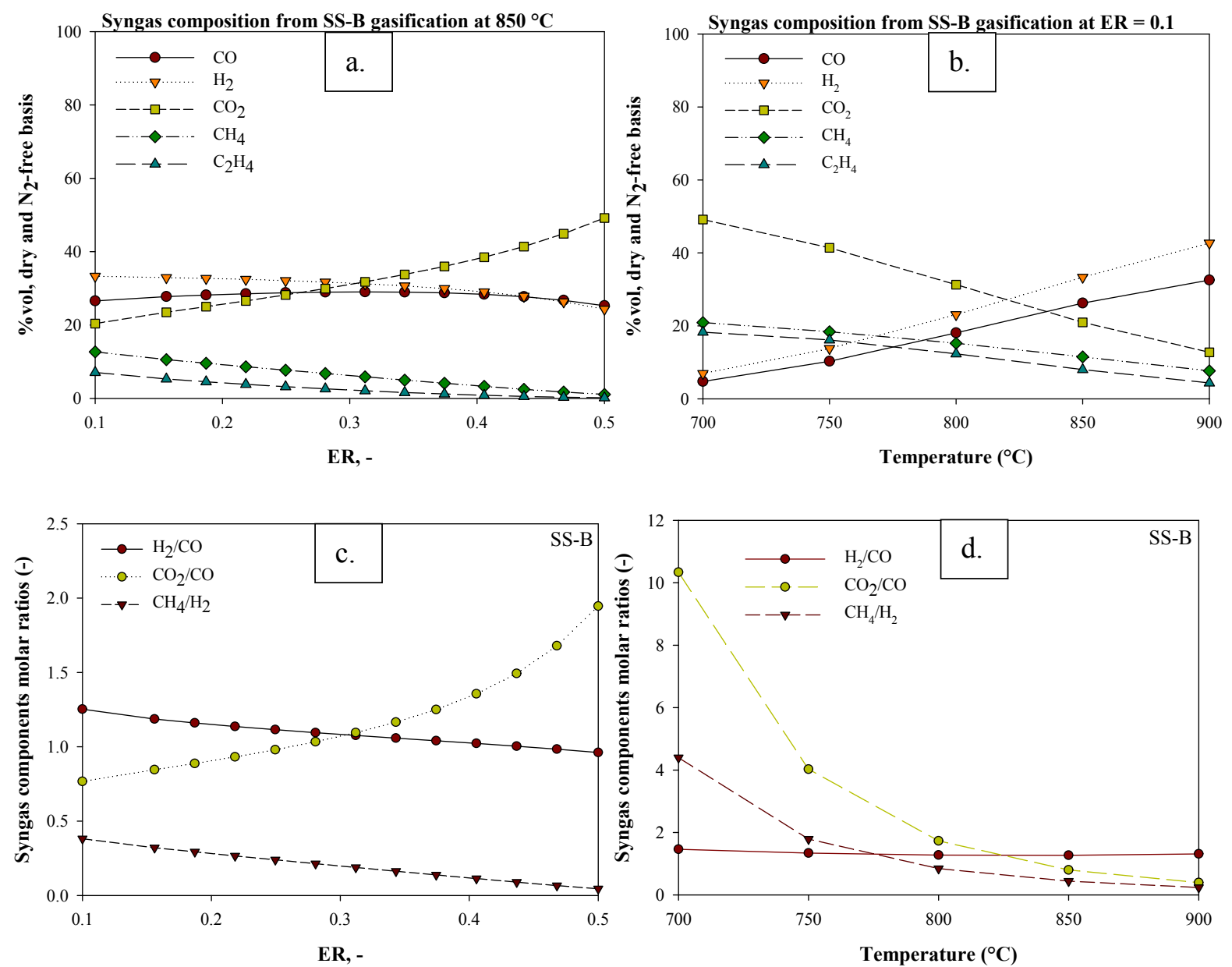

58

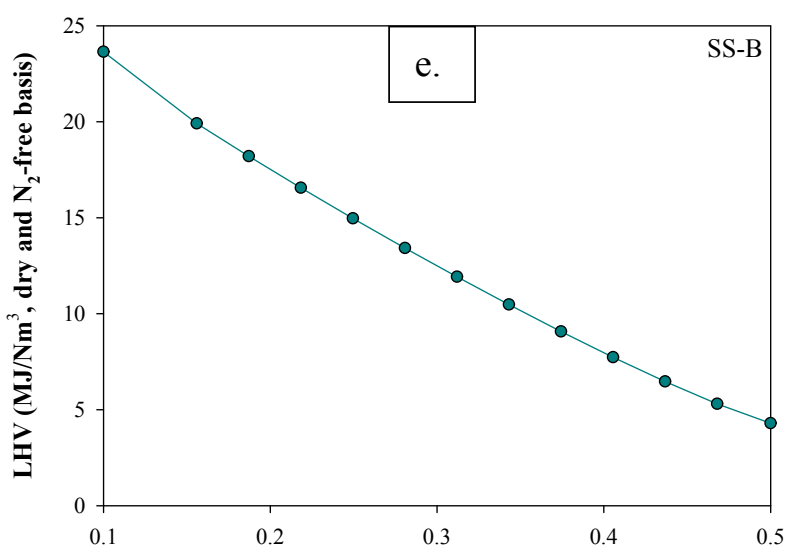

59

ER, -

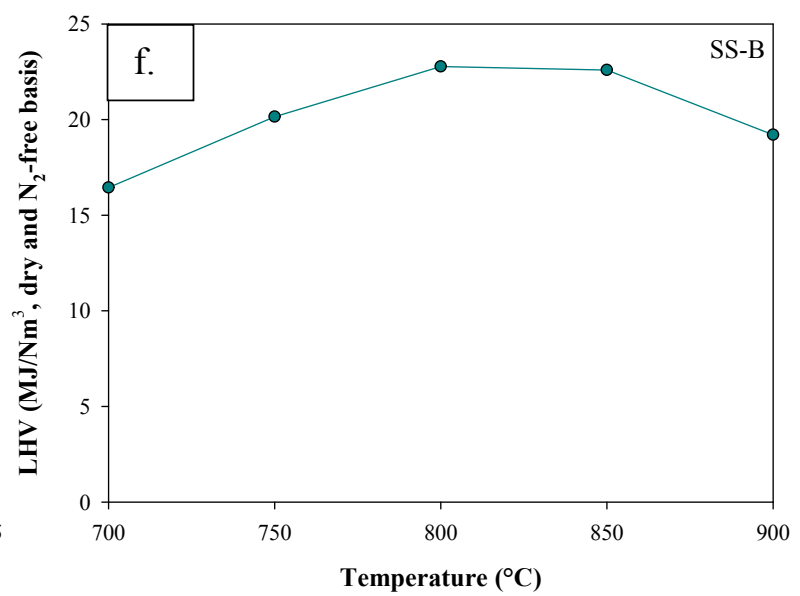

60 Figure S11. Simulated composition, molar ratios and LHV of syngas from gasification of SS-B

61 at different values of equivalence ratio (a, c and e, respectively, at $850{ }^{\circ} \mathrm{C}$ ) and reactor temperature ( $b, d$ and $f$, respectively, at $E R=0.1$ ). 


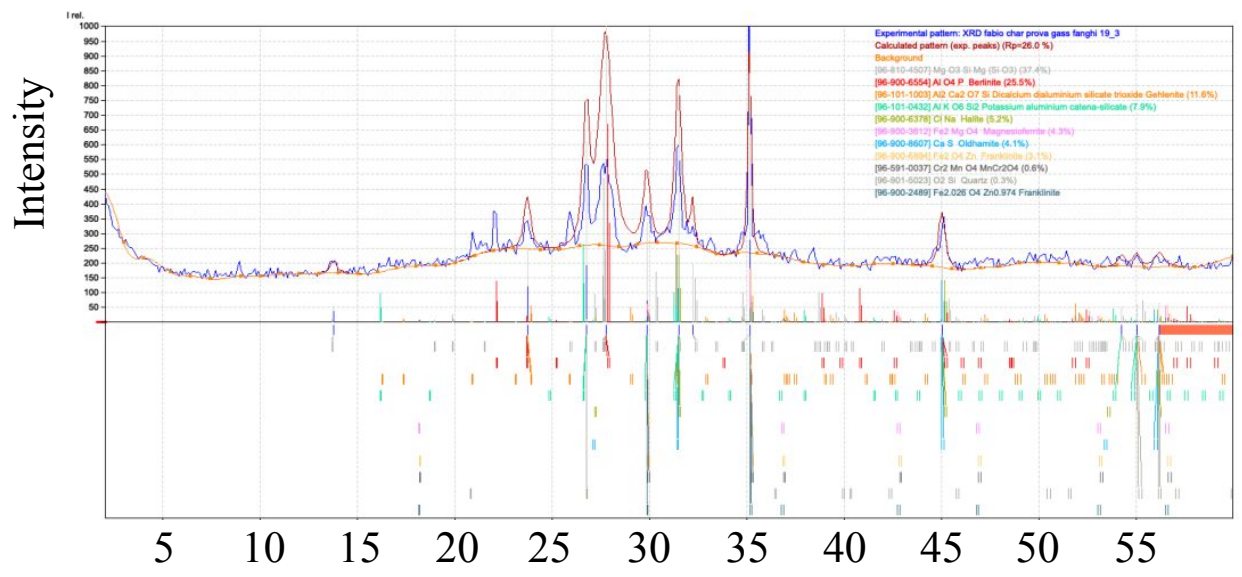

Diffraction angle, ${ }^{\circ} 2 \theta-\mathrm{Cu} \mathrm{k} \alpha$

\begin{tabular}{|c|l|ll|c|} 
Color & \multicolumn{1}{|c|}{ Entry } & \multicolumn{1}{|c|}{ Formula } & \multicolumn{1}{c|}{ Matched phase } & Quant.(\%) \\
\hline & $96-810-4507$ & Mg O3 SI & Mg (SI 03) & 37.4 \\
$96-900-6554$ & Al O4 P & Berlinite & 25.5 \\
$96-101-1003$ & Al2 Ca2 O7 SI & Dicalclum dialuminium sillicate tri... & 11.6 \\
$96-101-0432$ & Al K O6 SI2 & Potassium aluminium catena-slic... & 7.9 \\
$96-900-6378$ & Cl Na & Halite & 5.2 \\
$96-900-3612$ & Fe2 Mg O4 & Magnesioferrite & 4.3 \\
$96-900-8607$ & Ca S & Oldhamite & 4.1 \\
$96-900-6894$ & Fe2 O4 Zn & Franklinite & 3.1 \\
$96-591-0037$ & Cr2 Mn O4 & MnCr2O4 & 0.6 \\
$96-901-5023$ & O2 SI & Quartz & 0.3 \\
\hline
\end{tabular}

65 Figure S12. XRD spectrum for bottom ash from fluidized bed gasification of SS-A, with semiquantitative amounts for crystalline phases.

67 


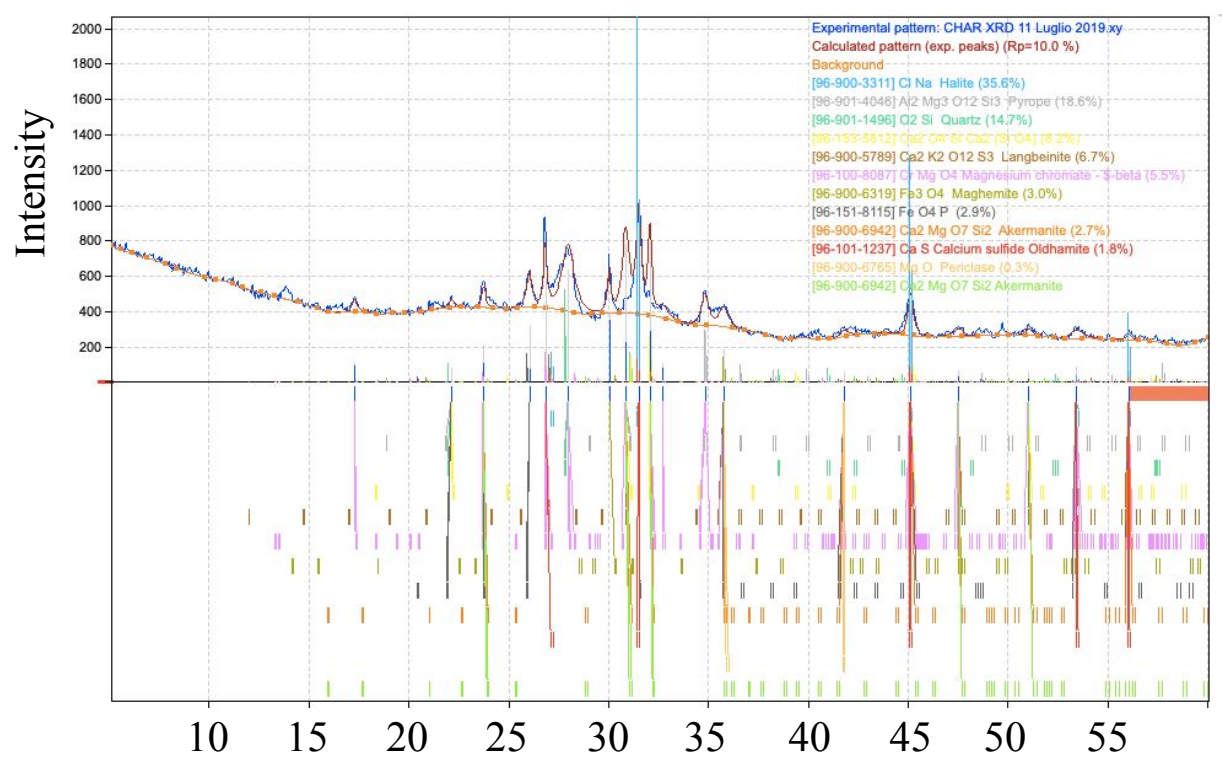

Diffraction angle, ${ }^{\circ} 2 \theta-\mathrm{Cu} \mathrm{k} \alpha$

\begin{tabular}{|c|c|c|c|c|}
\hline Color & Entry & Formula & Matched phase & Quant.(\%) \\
\hline & 96-900-3311 & $\mathrm{ClNa}$ & Halite & 35.6 \\
\hline & $96-901-4046$ & Al2 Mg3 $012 \mathrm{Si} 3$ & Pyrope & 18.6 \\
\hline & 96-901-1496 & $02 \mathrm{Si}$ & Quartz & 14.7 \\
\hline & $96-153-5812$ & $\mathrm{Ca} 204 \mathrm{Si}$ & $\mathrm{Ca} 2(\mathrm{Si}$ 04) & 8.2 \\
\hline & $96-900-5789$ & $\mathrm{Ca} 2 \mathrm{~K} 2012 \mathrm{~S} 3$ & Langbeinite & 6.7 \\
\hline & $96-100-8087$ & $\mathrm{CrMgO}$ & Magnesium chromate - \$-beta & 5.5 \\
\hline & $96-900-6319$ & $\mathrm{Fe} 304$ & Maghemite & 3.0 \\
\hline & $96-151-8115$ & $\mathrm{Fe} 04 \mathrm{P}$ & & 2.9 \\
\hline & $96-900-6942$ & $\mathrm{Ca} 2 \mathrm{Mg} 07 \mathrm{Si} 2$ & Akermanite & 2.7 \\
\hline & 96-101-1237 & Cas & Calcium sulfide Oldhamite & 1.8 \\
\hline & $96-900-6765$ & $\mathrm{MgO}$ & Periclase & 0.3 \\
\hline
\end{tabular}

69 Figure S13. XRD spectrum for bottom ash from fluidized bed gasification of SS-B, with semiquantitative amounts for crystalline phases.

71 

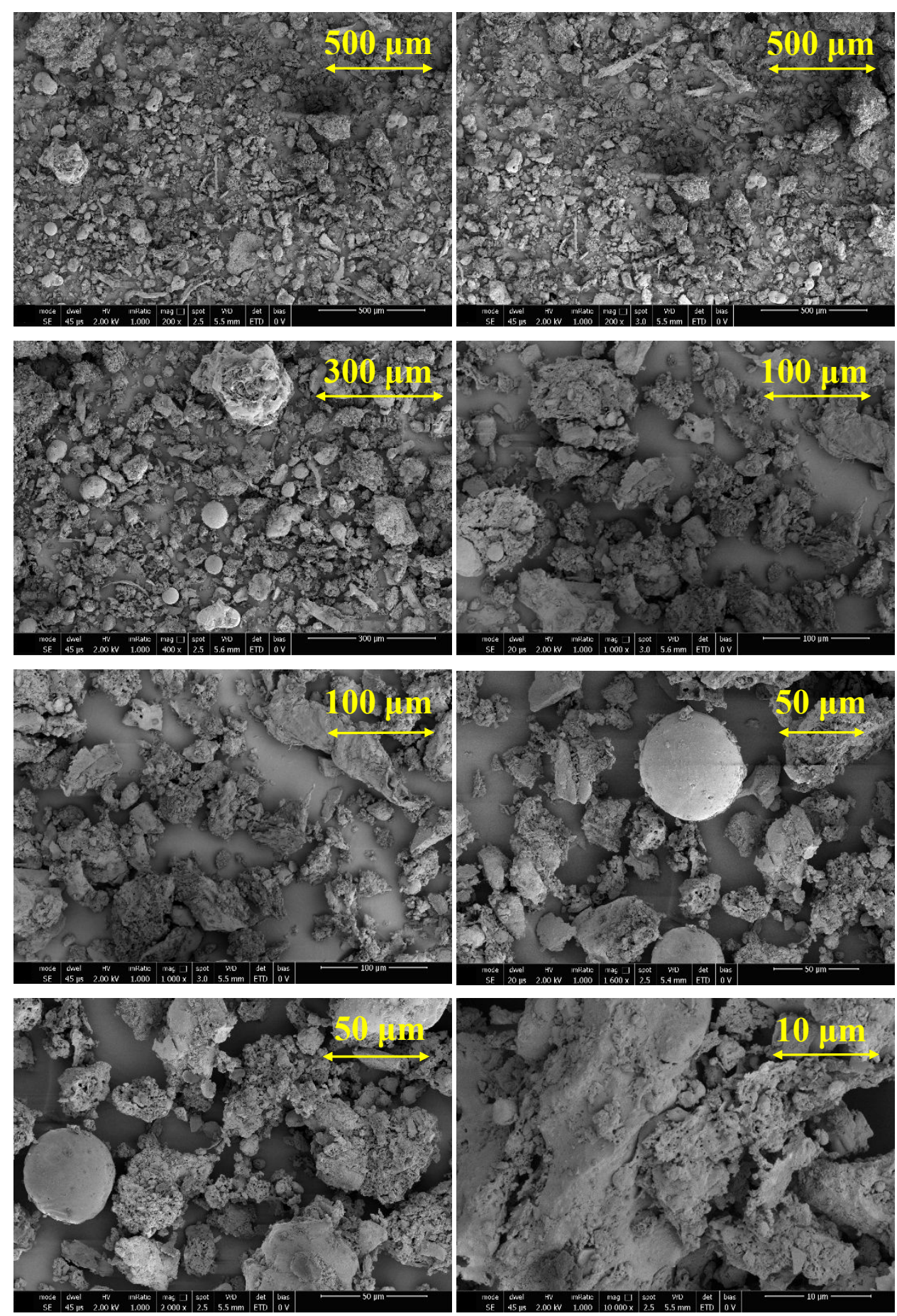

74 Figure S14. SEM images of bottom ash from fluidized bed gasification of SS-A (at increasing

magnification, from 200 up to $10000 \times$ ). 

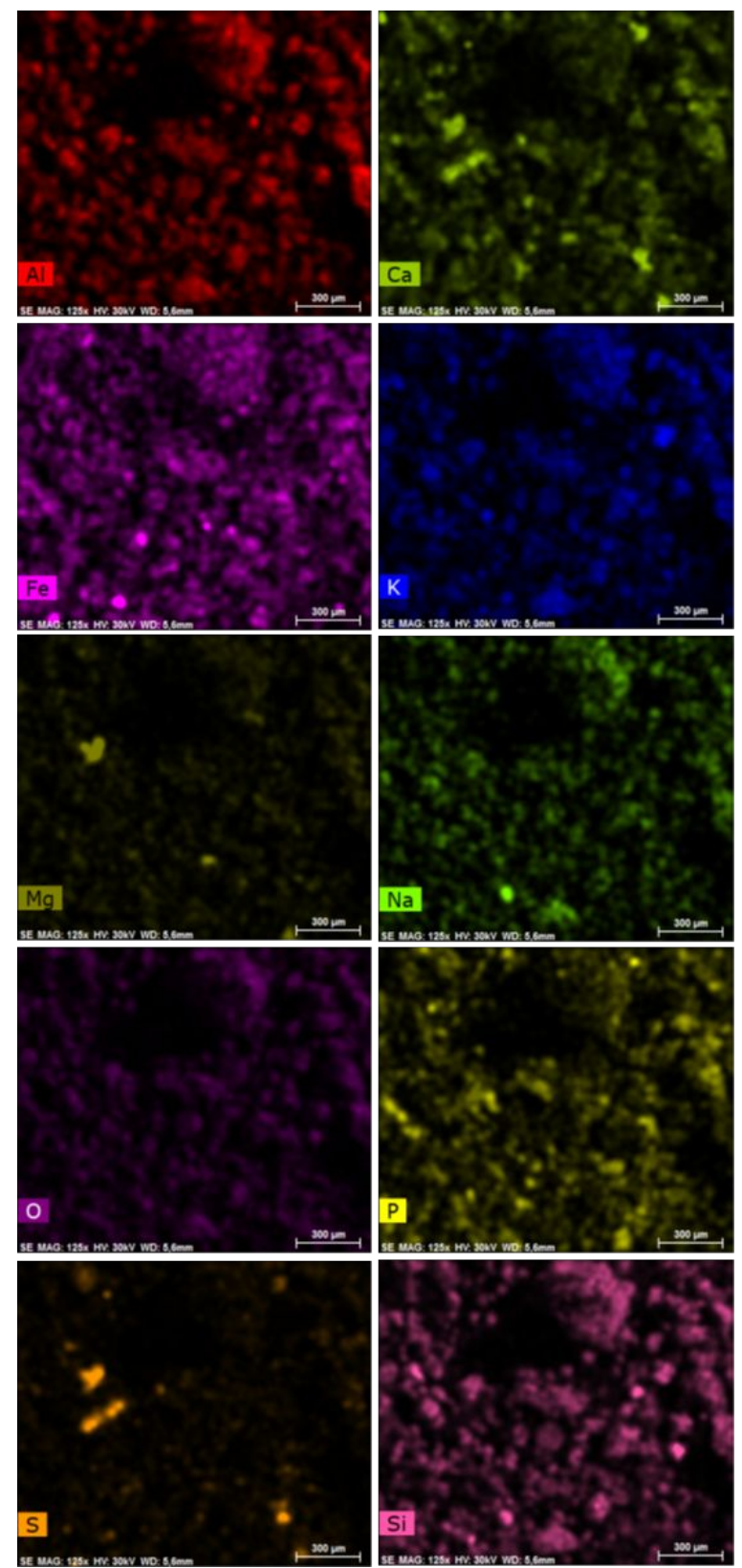

78 Figure S15. Distribution of single elements detected in bottom ash from fluidized bed 79 gasification of SS-A (sample of Figure 7 in the Manuscript) by EDX analysis. Position and 80 concentration of each element are identified by false colors and their intensity. 